\title{
Transition in household energy and impacts on regional air quality
}

\author{
GUOFENG SHEN $^{1}, \mathrm{XI}_{\text {ZHU }}^{2}$, WEI DU ${ }^{3}, \mathrm{SHU} \mathrm{TAO}^{4}$
}

${ }^{1}$ College of urban and environmental sciences, Peking

University, gfshen12@pku.edu.cn

${ }^{2}$ College of urban and environmental sciences, Peking University, zgczzx@foxmail.com

${ }^{3}$ School of geographic science, East China Normal

University,wdu@geo.ecnu.edu.cn

${ }^{4}$ College of urban and environmental sciences, Peking

University, taos@pku.edu.cn

Hosuehold energy consumption comprises up to a relatively smaller part of the total energy consumption in the society, however, in most developing countries, due to relatively low efficiencies, air pollutants such as primary PM2.5 and black carbon from this sector contributes significantly to the total emission, and adverse impacts on human health as the residential emissions influence outdoor air quality but also indoor air quality. Based on the first-hand data from national surveys and questionnaires, we analyzed patterns of household cooking and heating energies in rural mainland China, and transitions in household energy consumption over the 25 years from the 1990s. The results showed fast transitions in both cooking and heating energies, with increased use of clean fuels and electricity. Stacked use of different energies is common with more households had two or three energies in their daily lives. The contribution of residential emissions to outdoor air quality was quantified based on the updated emission inventory (PKU series) and regional air quality modelling. Rural residential emissions affected not only rural, but significantly to the urban air quality. Generally the contribution of residential emissions to outdoor air quality was decreasing, resulting in obvious cobenefits in human health and regional climate impacts (radiative forcing). However the serious issue is its direct impacts on indoor air quality, and high contributions to the overall exposure when taking both indoor and outdoor exposure into account. A systematic literature review revealed high rural air pollution in mainland China, due to high reliance on traditional solid fuels. Field campaigns were taken to characterize household air pollution and personal exposure by using personal carried samplers. Personal daily exposure level of PM2.5 was several times of that set by the WHO to protect human health, and a high fractions of submicron PM1.0 in inhaled particles were revealed. 